\title{
Neuroprem: the Neuro-developmental outcome of very low birth weight infants in an Italian region
}

Licia Lugli $i^{*}$ (D, Marisa Pugliese ${ }^{1}$, Carlotta Plessi ${ }^{1}$, Alberto Berardi ${ }^{1}$, Isotta Guidotti ${ }^{1}$, Gina Ancora ${ }^{2}$, Sara Grandi ${ }^{2}$, Giancarlo Gargano ${ }^{3}$, Silvia Braibanti ${ }^{3}$, Fabrizio Sandri ${ }^{4}$, Silvia Soffritti ${ }^{4}$, Elisa Ballardini ${ }^{5}$, Vittoria Arena ${ }^{5}$, Marcello Stella ${ }^{6}$, Serafina Perrone ${ }^{7}$, Sabrina Moretti ${ }^{7}$, Vittoria Rizzo ${ }^{6}$, Fabrizio Ferrari ${ }^{1}$, Neuroprem Working Group, Odoardo Picciolini, Roberto Bellù, Daniela Turoli, Luigi Tommaso Corvaglia, Gianpaolo Garani, Vittoria Paoletti, Giacomo Biasucci, Augusto Biasini, Belinda Benenati, Paolo Stagi, Cristina Magnani, Sara Dallaglio, Elisa DellaCasa Muttini, Maria Federica Roversi, Luca Bedetti, Laura Lucaccioni, Natascia Bertoncelli and Alessandra Boncompagni

\begin{abstract}
Introduction: The survival of preterm babies has increased worldwide, but the risk of neuro-developmental disabilities remains high, which is of concern to both the public and professionals. The early identification of children at risk of neuro-developmental disabilities may increase access to intervention, potentially influencing the outcome.

Aims: Neuroprem is an area-based prospective cohort study on the neuro-developmental outcome of very low birth weight (VLBW) infants that aims to define severe functional disability at 2 years of age.

Methods: Surviving VLBW infants from an Italian network of 7 neonatal intensive care units (NICUs) were assessed for 24 months through the Griffiths Mental Developmental Scales (GMDS-R) or the Bayley Scales of Infant and Toddler Development (BSDI III) and neuro-functional evaluation according to the International Classification of Disability and Health (ICF-CY). The primary outcome measure was severe functional disability at 2 years of age, defined as cerebral palsy, a BSDI III cognitive composite score < 2 standard deviation (SD) or a GMDS-R global quotients score $<2$ SD, bilateral blindness or deafness.
\end{abstract}

Results: Among 211 surviving VLBW infants, 153 completed follow-up at 24 months (72.5\%). Thirteen patients (8.5\%) developed a severe functional disability, of whom 7 presented with cerebral palsy (overall rate of 4.5\%). Patients with cerebral palsy were all classified with ICF-CY scores of 3 or 4. BSDI III composite scores and GMDS-R subscales were significantly correlated with ICF-CY scores $(p<0.01)$.

Conclusion: Neuroprem represents an Italian network of NICUs aiming to work together to ensure preterm neurodevelopmental assessment. This study updates information on VLBW outcomes in an Italian region, showing a rate of cerebral palsy and major developmental disabilities in line with or even lower than those of similar international studies. Therefore, Neuroprem provides encouraging data on VLBW neurological outcomes and supports the implementation of a preterm follow-up programme from a national network perspective.

Keywords: Neuro-developmental outcome, Very low birth weight preterm, Italy

\footnotetext{
*Correspondence: licia.lugli@virgilio.it; lugli.licia@policlinico.mo.it

${ }^{1}$ Neonatal Intensive Care Unit, University Hospital of Modena, Via del Pozzo

71, 41100 Modena, Italy

Full list of author information is available at the end of the article
}

(c) The Author(s). 2020 Open Access This article is distributed under the terms of the Creative Commons Attribution 4.0 International License (http://creativecommons.org/licenses/by/4.0/), which permits unrestricted use, distribution, and reproduction in any medium, provided you give appropriate credit to the original author(s) and the source, provide a link to the Creative Commons license, and indicate if changes were made. The Creative Commons Public Domain Dedication waiver (http://creativecommons.org/publicdomain/zero/1.0/) applies to the data made available in this article, unless otherwise stated. 


\section{Introduction}

The survival of preterm babies has increased worldwide, with a concomitant decrease in severe neonatal morbidity. However, the risk of neuro-developmental and behavioural disabilities remains high in children and adults who are born preterm. The high rates of neurological and developmental problems reported in survivors are of concern to both the public and professionals. Among extremely preterm babies (22-26 weeks gestation), reported survival without neuro-developmental impairment at 2 years is variable, from 20 to $42 \%$ [1-3]. In absolute numbers, infants born very and moderately preterm represent a larger proportion of preterm births, accounting for more children with motor, cognitive, or behavioural deficits and learning disabilities [4-6]. The early identification of children at risk of neuro-developmental disabilities may increase access to intervention, potentially improving the outcome [7]. The Bayley Scales of Infant and Toddler Development and the Griffith Mental Developmental Scale are used for neuro-developmental evaluation between 18 and 30 months, depending on the local follow-up protocols [8-10]. Both are costly, time consuming, and require trained staff. Alternative tools that can effectively screen for developmental delay in a uniform way are therefore needed. Neuro-functional evaluation according to the International Classification of Disability and Health is accepted by the World Health Organization (WHO) as a valid developmental screening tool and is becoming increasingly popular to assess development [11]. The International Classification of Functioning, Disability, and Health - Children and Youth version (ICF-CY) (World Health Organization, 2007) endorses the biopsychosocial model and comprehensively addresses disability by linking neuro-developmental outcomes to social and environmental factors [11]. Different studies have shown the value of ICF-CY-based datasets to compare functioning and disability data in children of different ages [12, 13]. Moreover, the ICF-CY-based approach has been implemented successfully in routine follow-up programmes for preterm infants $[14,15]$. It is easy to administer and interpret; it has a short completion time and it can decrease data heterogeneity. It enables clinicians to focus on those children suspected of having developmental delay and hence needing further developmental assessment or intervention.

Updated data on the neuro-development of very low birth weight (VLBW) infants are scant, and national networks on preterm neurological outcomes are still lacking in Italy.

This is an area-based prospective cohort study on the neuro-developmental outcome of preterm infants that aims to define severe functional disability at 2 years of corrected age among VLBW infants in an Italian region. For this purpose, different neuro-developmental evaluation instruments (the Bayley Scales of Infant and
Toddler Development, BSDI III; the Griffith Mental Developmental Scale, GMDS-R; and the ICF-CY) were used and compared.

\section{Methods}

Neuroprem represents an Italian network of NICUs aiming to work together to ensure preterm neurodevelopmental assessment. In 1999, the NICUs of an Italian region (Emilia Romagna) joined the Vermont Oxford Network Database [16] and in 2015-2016 set up this prospective cohort study on the neuro-developmental outcome of VLBW infants. Before starting patient enrolment, NICUs participated in seminars and meetings to define and share the study protocol. A common data collection form was created, including perinatal and neurodevelopmental follow-up data. Anonymized data were collected through a web platform. For the purpose of this study, the cohort of VLBW infants born in 2016 was enrolled, and their neuro-developmental data at 24 months, corrected for prematurity, were collected. Surviving infants were assessed with neurological examination according to the Amiel-Tison neurological assessment [17] and either with the Griffiths Mental Developmental Scales (GMDS-R, 1996) (18) or the Bayley Scales of Infant and Toddler Development (BSDI III, 2006) [18], depending on the local protocols.

GMDS-R (0-2 years) provides a General Development Quotient (GQ) of infants' abilities with a mean of 100.5 and an SD of 11.8 and five subscale quotients (Locomotor, Eye \& Hand Coordination, Personal \& Social, Hearing \& Language, and Cognitive Performance) [19]. The BSDI III provides standardized composite scores for each of the assessed domains (cognitive, fine and gross motor, receptive and expressive language, and adaptive), with a mean of 100 and an SD of 15 [18]. For both the GMDS-R and BSID-III, the cut-off abnormality was 2 standard deviations (SD) below the normative mean. BSDI III or GMDS-R results below 2 SD were compared among 3 groups of different gestational ages.

Enrolled patients were also assessed with neurofunctional evaluation according to the International Classification of Disability and Health (ICF-CY) [11]. Neuro-functional clinical evaluation was performed for cognitive, linguistic, motor, and adaptive function, and then a global score ICF-CY was assigned (Appendix).

The rate and type of cerebral palsy were evaluated [20].

The primary outcome measure was severe functional disability at 2 years of age, corrected for prematurity. Severe functional disability was defined as follows: cerebral palsy, a BSDI III cognitive composite score $<2$ SD or a GMDS-R GQ < 2 SD, bilateral blindness (visual acuity < $6 / 60$ in better eye), or bilateral deafness (requiring bilateral hearing aids or unilateral/bilateral cochlear implants). 
The correlation between ICF-CY, BSDI III or GMDS$\mathrm{R}$ and cerebral palsy was evaluated.

The study was approved by the Ethical Committee. For every enrolled patient, written consent was signed by the parents.

Statistical analyses were performed using Stata Direct Statistical Software version 13 (StataCorp LP, USA). Continuous variables were described using means and $\mathrm{SD}$, while categorical variables were described using frequencies. Groups were compared by $x^{2}$ analyses for categorical variables and by analysis of variance for continuous data. Agreement between different tests was evaluated with kappa coefficient. A $p$ value $<0.05$ was considered statistically significant.

\section{Results}

A total of 228 VLBW infants were enrolled from 7 NICUs of the Emilia Romagna region (all tertiary referral centers). Seventeen died (7.4\%). Among 211 survivors, 153 patients completed follow-up at 24 months (72.5\%). They had gestational ages between 23 and 33 weeks (mean gestational age 29.1 \pm 2.8 ; CI 28.6-29.5) and birth weights between $495 \mathrm{~g}$ and $1500 \mathrm{~g}$ (mean birth weight $1113.9 \mathrm{~g} \pm 283.5$; CI 1068.7-1159.2). No differences in gestational age, birth weight, gender, ethnicity, or parental education were found between patients who completed follow-up and patients who dropped out. These 153 patients included in the research received mechanical ventilation for a mean duration of $4.4 \pm 11$.4 days, non invasive ventilation for $19.0 \pm 23.2$ days and oxygen supplementation for $25.6 \pm 33.8$ days. Mean hospital stay was $54.3 \pm 31.2$ days and mean body weight at discharge from hospital was $2231.7 \mathrm{~g} \pm 518.4$. After discharge 31/ 153 (20.3\%) infants were included in a program of neuro-motor rehabilitation.
Among 153 patients completing follow-up, 86 (56.2\%) were evaluated with the BDS-III and 67 (43.8\%) with the GMDS-R. Table 1 shows the BSDI III and GMDS-R results among the 3 different gestational age groups. Cognitive function evaluated with the ICF-Y score was significantly worse in the lower gestational age group, while motor, language and adaptive function were not significantly different among the 3 groups (Table 2). The rate of patients classified with an ICF-CY score $\geq 2$ was significantly higher in the lower gestational age group: 14/54 $(25.9 \%)$ in preterm infants with $\leq 28$ weeks gestational age; 9/58 (15.5\%) in patients with 29-30 weeks gestational age; and $2 / 41(4.9 \%)$ in patients with $\geq 31$ weeks gestational age (comparison among 3 groups, $p=0.0224$ ).

BSDI III composite scores and GMDS-R subscales were significantly correlated with ICF-CY cognitive, motor, language, and adaptive function $(p<0.01)$. The agreement between BSDI III composite scores and ICF-CY was 95.3\% (weighted Kappa $=0.84$ ) for cognitive domain and 95.3\% (weighted Kappa =0.86) for motor domain. The agreement between GMDS-R were and ICF-CY was 88.1\% (weighted Kappa $=0.76$ ) for cognitive performance and $92.5 \%$ (weighted Kappa $=0.75$ ) for locomotor subscales.

Thirteen (8.5\%) patients were classified with severe functional disability: 6 showed a $<2$ SD GQ score and 7 had cerebral palsy (cerebral palsy overall rate of $4.5 \%$ ) (Table 3). None of the patients had severe vision deficits or deafness. Among patients with cerebral palsy, three had tetraplegia, three had diplegia and one had hemiplegia. Four cases of cerebral palsy occurred in patients with $\leq 28$ weeks gestational age (7.4\% rate), 1 case of cerebral palsy occurred among patients with 29-30 weeks gestational age (1.7\% rate), and 2 cases of cerebral palsy occurred among patients with $\geq 31$ weeks gestational age (4.8\%), which was not significantly different among groups.

Table 1 BSDI-III or GMDS-R evaluation and comparison among different gestational age groups

\begin{tabular}{|c|c|c|c|c|c|}
\hline & All Patients (153) & GA $\leq 28$ weeks $(54)$ & GA 29-30 weeks (58) & $\mathrm{GA} \geq 31$ weeks (41) & P \\
\hline Patients evaluated with GMDS-R (\%) & $67 / 153(438)$ & $20 / 54(37)$ & $26 / 58(44.8)$ & $21 / 48(51.2)$ & - \\
\hline GMDS-R performance subquotient $<2$ SD & $6 / 67(8.9)$ & $3 / 20(15)$ & $3 / 26(11.5)$ & $0 / 21(0)$ & 0.2045 \\
\hline GMDS-R locomotor subquotient $<2$ SD & 2/67 (3) & $1 / 20(5)$ & $0 / 26(0)$ & $1 / 21(4.8)$ & 0.5196 \\
\hline GMDS-R hearing and language subquotient $<2$ SD & $5 / 67(7.4)$ & $3 / 20(15)$ & $2 / 26(7.7)$ & $0 / 21(0)$ & 0.1882 \\
\hline GMDS-R personal \& social subquotient $<2$ SD & $1 / 67(15)$ & $1 / 20(5)$ & $0 / 26(0)$ & $0 / 21(0)$ & 0.3034 \\
\hline GMDS-R Eye \& Hand Coordination subquotient $<2$ SD & $5 / 67(7.5)$ & $3 / 20(15)$ & $2 / 26(7.7)$ & $0 / 21(0)$ & 0.1381 \\
\hline GMDS-R global quotient $<2$ SD & 6/67 (8.9) & $3 / 20(15)$ & $3 / 26(11,5)$ & $0 / 21(0)$ & 0.2045 \\
\hline Patients evaluated with BSDI-III (\%) & $86 / 153(56.2)$ & $34 / 54(63)$ & $32 / 58(55.2)$ & 20/41 (48.8) & - \\
\hline BSDI-III Cognitive composite score $<2$ SD & $3 / 86(3.5)$ & 3/34 (8.8) & $0 / 32(0)$ & $0 / 20(0)$ & 0.0928 \\
\hline BSDI-III Motor composite score < 2SD & 4/86 (4.6) & 3/34 (8.8) & $0 / 32(0)$ & $1 / 20(5)$ & 0.2344 \\
\hline BSDI-III Language composite score $<2$ SD & 8/86 (9.3) & 6/34 (17.6) & $1 / 32(3.1)$ & $1 / 20(5)$ & 0.0957 \\
\hline BSDI-III Adaptive composite score $<2$ SD & $8 / 86(9.3)$ & $5 / 34(14.7)$ & $2 / 32(6.2)$ & $1 / 20(5)$ & 0.3737 \\
\hline
\end{tabular}


Table 2 Neuro-functional clinical evaluation (ICF-CY) and comparison among different gestational age groups

\begin{tabular}{|c|c|c|c|c|c|}
\hline & All patients (\%) & $\mathrm{GA} \leq 28$ weeks $(\%)$ & GA 29-30 weeks (\%) & GE $\geq 31$ weeks (\%) & $P$ \\
\hline Number (\%) & $153(100)$ & $54(35.2)$ & $58(38)$ & $41(26.8)$ & - \\
\hline \multicolumn{6}{|c|}{ Cognitive function } \\
\hline 1 & $128(83.7)$ & $39(72.2)$ & $50(86.2)$ & $39(95.1)$ & \multirow[t]{3}{*}{0.0414} \\
\hline 2 & 18 (11.6) & $11(20.4)$ & $5(8.6)$ & $2(4.9)$ & \\
\hline 3 & $7(4.6)$ & $4(7.4)$ & $3(5.2)$ & $0(0)$ & \\
\hline \multicolumn{6}{|c|}{ Motor function } \\
\hline 1 & $132(6.3)$ & $43(79.6)$ & $52(89.7)$ & $37(90.2)$ & \multirow[t]{3}{*}{0.3917} \\
\hline 2 & $14(9.1)$ & $7(13)$ & $5(8.6)$ & $2(4.8)$ & \\
\hline 3 & $7(4.6)$ & $4(7.4)$ & $1(1.7)$ & $2(4.8)$ & \\
\hline \multicolumn{6}{|c|}{ Language function } \\
\hline 1 & $110(71.9)$ & $36(66.7)$ & $43(74.1)$ & $31(75.6)$ & \multirow[t]{3}{*}{0.166} \\
\hline 2 & $34(22.2)$ & $13(24)$ & $11(19)$ & $10(24.3)$ & \\
\hline 3 & $9(5.9)$ & $5(9.3)$ & $4(6.9)$ & $0(0)$ & \\
\hline \multicolumn{6}{|c|}{ Adaptive function } \\
\hline 1 & $138(90.1)$ & $47(87.1)$ & $52(89.7)$ & $39(95.2)$ & \multirow[t]{4}{*}{0.6328} \\
\hline 2 & $9(5.9)$ & $4(7.4)$ & $4(6.9)$ & $1(2.4)$ & \\
\hline 3 & $5(3.3)$ & $3(5.5)$ & $1(1.7)$ & $1(2.4)$ & \\
\hline 4 & $1(0.7)$ & $0(0)$ & $1(1.7)$ & $0(0)$ & \\
\hline \multicolumn{6}{|l|}{ ICF-CY } \\
\hline 0 & $99(64.7)$ & $30(55.6)$ & $40(69)$ & $29(70.7)$ & \multirow[t]{5}{*}{0.0910} \\
\hline 1 & $29(18.9)$ & $10(18.5)$ & $9(15.5)$ & $10(24.4)$ & \\
\hline 2 & $18(11.8)$ & $10(18.5)$ & $8(13.8)$ & $0(0)$ & \\
\hline 3 & $5(3.3)$ & $2(3.7)$ & $1(1.7)$ & $2(4.9)$ & \\
\hline 4 & $2(1.3)$ & $2(3.7)$ & $0(0)$ & $0(0)$ & \\
\hline
\end{tabular}

GA gestational age

Table 3 Patients with severe functional disabilities

\begin{tabular}{lllll}
\hline Case & Gestational age (weeks) & Weight (gr) & Severe functional disability & ICF-CY \\
\hline 1 & 30 & 1080 & Tetraplegia & 3 \\
2 & 34 & 1480 & Diplegia & 3 \\
3 & 23 & 550 & Tetraplegia, BSDI-III cognitive composite score $<2$ DS & 4 \\
4 & 31 & 1290 & Diplegia & 3 \\
5 & 27 & 730 & GMDS-R global quotient $<2$ DS & 2 \\
6 & 29 & 1175 & GMDS-R global quotient $<2$ DS & 2 \\
7 & 28 & 1100 & GMDS-R global quotient $<2$ DS & 2 \\
8 & 29 & 930 & GMDS-R global quotient $<2$ DS & 2 \\
9 & 30 & 1130 & GMDS-R global quotient $<2$ DS & 2 \\
10 & 28 & 700 & GMDS-R global quotient $<2$ DS & 4 \\
11 & 26 & 1050 & Tetraplegia, BSDI-III cognitive composite score $<2$ DS \\
12 & 28 & 1248 & Hemiplegia, BSDI-III cognitive composite score $<2$ DS & 3 \\
13 & 24 & 690 & Diplegia & 3 \\
\hline
\end{tabular}


Among 13 patients with severe functional disability, 6 children were classified with an ICF-CY score of 2 (46.1\%), 5 with an ICF-CY score of $3(38.5 \%)$ and 2 with an ICF-CY score of 4 (15.4\%). Patients with cerebral palsy were all classified with ICF-CY scores of 3 or 4 (Table 3).

There was no significant correlation between invasive/ non-invasive ventilation, oxygen supplementation or hospital stay duration and outcome.

\section{Discussion}

This is an Italian area-based study on the neurodevelopmental outcome of VLBW preterm infants. In this cohort prospective study, the rate of severe functional disability was $8.5 \%$, and the rate of cerebral palsy was $4.5 \%$. Other follow-up studies revealed a higher prevalence of developmental disabilities in very preterm neonates, as cerebral palsy was previously reported in $10-15 \%$ of cases [21-25]. It could be claimed that our encouraging results depend on a selection bias due to the follow-up dropout rate. The group of patients completing the 24-month follow-up (72.5\%) and the group who was lost to followup were comparable for gestational age, birth weight, ethnicity and parental education. Therefore, there was no additional known risk factor for neuro-developmental disabilities in the group lost to follow-up; moreover, the follow-up dropout rate we found was similar to that reported in previous studies. The lower rate of developmental disabilities of our study probably addresses differences in the selected study population. While most of the previous studies focused on extremely or very preterm newborns, in our study, we included VLBW infants, among whom gestational age is extremely variable (from 23 to 33 weeks of gestation). VLBW preterm infants have been poorly investigated in the past, but knowledge of their neuro-developmental trajectory could lead to targeted intervention and prevention of later disabilities, as timely intervention has a positive influence on cognitive outcome in infancy $[1-3,6]$. Indeed, in the Neuroprem study a considerable number of patients was included in a program of neuro-motor rehabilitation, precisely because of prematurity risk factor or because of early sign of neurological abnormalities. Probably their neurodevelopmental outcome was influenced by the precocity of these interventions. On the other hand, we have not found any correlation between ventilation, oxygen supplementation or hospital stay duration and outcome, but further larger studies focusing on the effect of perinatal factors on outcome are desirable.

We have insufficient data to evaluate the impact of the etiology of the VLBW on neuro-developmental outcome, but in our study the rates of cerebral palsy did not differ significantly among gestational ages, and cerebral palsy occurred even in preterm infants with $\geq 31$ weeks of gestation. Additionally, the BSDI III and GMDS-R results were comparable in patients with different gestational ages, but the small sample size may have contributed to defeat the statistical analysis. In contrast, cognitive function, evaluated with the ICF-CY in the whole study population, was significantly worse in the lower gestational age group, while motor, language and adaptive function were not significantly different among groups. This means that very and extremely preterm infants are at higher risk of neuro-developmental disabilities, but moderately preterm infants may also have neurological abnormalities $[4,6,26]$. Considering these results, a follow-up programme should also include moderately preterm infants and at least VLBW infants. The strength of our research is the prospective and areabased design of the study. Neuroprem created a network of Italian NICUs, adopting a definite follow-up programme for VLBW neuro-developmental outcome assessment. Such neonatal follow-up networks may contribute to guiding health policies and increase access to formal neuro-developmental evaluation and subsequent rehabilitative interventions [7, 24]. Actually, developmental delay rather than survival is recognized as the main problem in children born preterm $[1-3,6]$. The early identification of children at risk of later developmental difficulties may increase access to intervention, potentially influencing the course of otherwise persistent difficulties.

The ICF-CY-based approach has been implemented in follow-up programmes for preterm infants; it is a valid neuro-developmental screening and leads to overcoming dataset heterogeneity due to the local protocol of evaluation $[14,15]$. It allows clinicians to focus on those children suspected of having a developmental delay and hence needing further developmental assessment or rehabilitation. In our study, ICF-CY subscales significantly correlated with BSDI III composite scores and GMDS-R subscales and their agreement was high. Moreover, the ICF-CY permitted the recognition of all patients with cerebral palsy or severe functional disability, leading to a targeted intervention.

The current study has some limitations. First, the study population is small, and the enrolment was performed for only one year, which may not be representative of the trend over years. Second, the follow-up is limited to the first 2 years of life, and only severe functional disabilities were investigated; therefore, we did not evaluate mild neurological dysfunction and preschool age performance, which may be impaired in different neuropsychological domains even in patients without major disabilities [5,21,27]. Nevertheless, this study is a first attempt to highlight the status of VLBW outcomes in an Italian region. Third, developmental quotient data are heterogeneous because either the BSD-III or the 
GMDS-R was used, depending on the local protocol. The standardization of developmental tests among centres is desirable, but it requires staff training and is costly and time consuming. To overcome data heterogeneity coming from the BSD-III and GMDS-R, ICF-CY was adopted by all centres, and a good correlation between the ICF-CY, BSD-III and GMDS-R was achieved.

In conclusion, this study has contributed to updating information on outcomes for VLBW infants in an Italian region. Indeed, Neuroprem has started to build up an Italian network of NICUs that aim to work together to ensure VLBW neuro-developmental follow-up. Therefore, Neuroprem may represent a model for preterm follow-up programme implementation ad neuro-developmental outcome data collection.

\section{Conclusion}

Neuroprem represents an Italian network of NICUs aiming to work together to ensure preterm neuro-developmental assessment. We report on an Italian area-based prospective cohort study on the neuro-developmental outcome of very low birth weight (VLBW) preterm infants. This study updates information on VLBW outcomes in an Italian region, showing a rate of cerebral palsy and major developmental disabilities in line with or even lower than those of similar international studies. Therefore, Neuroprem provides encouraging data on VLBW neurological outcomes and supports the implementation of a preterm follow-up programme from a national network perspective.

\section{Supplementary information}

Supplementary information accompanies this paper at https://doi.org/10 1186/s13052-020-0787-7.

Additional file 1. Appendix A Neuro-functional clinical evaluation

\section{Acknowledgements}

The authors acknowledge the Mariani Foundation of Milan, who sustained and sponsored the study, and the personnel of all NICUs participating in the study.

\section{Authors' contributions}

-Licia Lugli gave substantial contributions to the conception of the study and drafted the work. -Marisa Pugliese contributed to the interpretation of data. -Carlotta Plessi contributed to the acquisition and analysis of data. -Alberto Berardi revised the paper. -Isotta Guidotti gave substantial contribution to the creation of the software used in the work. -Gina Ancora contributed to the design of the study and to interpretation of data. -Sara Grandi contributed to acquisition of data. -Giancarlo Gargano contributed to analysis of data and revision of the manuscript. -Silvia Braibanti contributed to acquisition of data. -Fabrizio Sandri contributed to analysis of data and revision of the manuscript. -Silvia Soffritti contributed to acquisition of data. -Elisa Ballardini contributed to analysis of data and interpretation of data. -Vittoria Arena contributed to acquisition of data. -Marcello Stella contributed to acquisition of data. -Serafina Perrone contributed to analysis of data and to revision of the paper. -Sabrina Moretti contributed to acquisition of data. -Vittoria Rizzo contributed to acquisition of data. -Fabrizio Ferrari gave substantial contributions to the design of the work and revised it. -Neuroprem Working Group contributed to the follow-up of patients. All Authors approved the submitted version and agreed to be personally accountable for the author's own contributions and to ensure that questions related to the accuracy or integrity of any part of the work are appropriately investigated and resolved.

\section{Funding}

this study was funded by Pier Franco and Luisa Mariani of Milan, awarding a grant to Licia Lugli.

\section{Availability of data and materials}

Data and materials are available for consultation in a web platform (REDCap).

Ethics approval and consent to participate

The study has been approved by the Ethical Committee of Emilia Romagna (number 4818).

\section{Consent for publication}

written consent was obtained for every enrolled patient. Consent copies are available if required.

\section{Competing interests}

the Authors declare that they have no competing interests.

\section{Author details}

${ }^{1}$ Neonatal Intensive Care Unit, University Hospital of Modena, Via del Pozzo 71, 41100 Modena, Italy. ${ }^{2}$ Neonatal Intensive Care Unit, Infermi Hospital of Rimini, Rimini, Italy. ${ }^{3}$ Neonatal Intensive Care Unit, Santa Maria Nuova Hospital of Reggio Emilia, Reggio Emilia, Italy. ${ }^{4}$ Neonatal Intensive Care Unit, Maggiore Hospital of Bologna, Bologna, Italy. ${ }^{5}$ Neonatal Intensive Care Unit, Sant'Anna Hospital of Ferrara, Sant'Anna, Italy. ${ }^{6}$ Neonatal Intensive Care Unit, Bufalini Hospital of Cesena, Cesena, Italy. ${ }^{7}$ Neonatal Intensive Care Unit, University Hospital of Parma, Parma, Italy.

Received: 14 August 2019 Accepted: 7 February 2020

Published online: 22 February 2020

\section{References}

1. Moore T, Hennessy EM, Myles J, et al. Neurological and developmental outcome in extremely preterm children born in England in 1995 and 2006: the EPICURE studies. BMJ. 2012;345:e7961.

2. Serenius F, Källén K, Blennow M, et al. EXPRESS group. Neurodevelopmental outcome in extremely preterm infants at 2.5 years after active perinatal care in Sweden. JAMA. 2013;309:181020.

3. Younge N, Goldstein RF, Bann CM, et al. Eunice Kennedy Shriver National Institute of Child Health and Human Development neonatal research network. Survival and neurodevelopmental outcomes among Periviable infants. N Engl J Med. 2017:376:617-28.

4. Larroque B, Ancel PY, Marret S, et al. EPIPAGE study group. Neurodevelopmental disabilities and special care of 5-yearold children born before 33 weeks of gestation (the EPIPAGE study): a longitudinal cohort study. Lancet. 2008;371:813-20.

5. Larroque B, Ancel PY, Marchand-Martin L, et al. Epipage study group. Special care and school difficulties in 8-year-old very preterm children: the Epipage cohort study. PLoS One. 2011;6:e21361.

6. Quigley MA, Poulsen G, Boyle E, et al. Early term and late preterm birth are associated with poorer school performance at age 5 years: a cohort study. Arch Dis Child Fetal Neonatal Ed. 2012;97:F167-73.

7. Van Wassenaer-Leemhuis AG, Jeukens-Visser M, van Hus JWP, et al. Rethinking preventive post-discharge intervention programmes for very preterm infants and their parents. Dev Med Child Neurol. 2016;58:67-73.

8. Benassi E, Savini S, Iverson JM, Guarini A, et al. Early communicative behaviors and their relationship to motor skills in extremely preterm infants. Res Dev Disabil. 2016;48:132-44.

9. Zuccarini M, Sansavini A, Iverson JM, et al. Object engagement and manipulation in extremely preterm and full term infants at 6 months of age Res Dev Disabil. 2016;55:173-84.

10. Sansavini A, Guarini A, Caselli MC. Preterm birth: neuropsychological profiles and atypical developmental pathways. Dev Disabil Res Rev. 2011;17(2):102-13.

11. World Health Organization (2007). The international classification of functioning, disability and health for children and youth: ICF-CY. Geneva, Switzerland: World Health Organization. 
12. Ibragimova N, Granlund M, Bjorck-Akesson E. Field trial of ICF version for children and youth (ICF-CY) in Sweden: logical coherence, developmental issues and clinical use. Dev Neurorehabil. 2009;12:3-11.

13. Meucci P, Leonardi M, Sala M, Martinuzzi A, et al. A survey on feasibility of ICF-CY use to describe persisting difficulties in executing tasks and activities of children and adolescent with disability in Italy. Disabil Health J. 2014;7: 433-41.

14. Giovannetti AM, Raggi A, Leonardi M, et al. Usefulness of ICF-CY to define functioning and disability: a retrospective study on children born with very low birth weight. Early Hum Dev. 2013;89:825-31.

15. Fontana C, Picciolini O, Fumagalli M, et al. A longitudinal ICF-CY-based evaluation of functioning and disability of children born with very low birth weight. Int J Rehabil Res. 2016;39:296-301.

16. Vermont Oxford Network. Vermont Oxford Database Manual of Operations for Infants born in 2009. Release 13.0: Burlington, VT: Vermont Oxford Network; 2008

17. Gosselin J, Gahagan S, Amiel-Tison C. The Amiel-Tison neurological assessment at term: conceptual and methodological continuity in the course of follow-up. Ment Retard Dev Disabil Res Rev. 2005;11:34-51.

18. Bayley N. The Bayley cales of infant and toddler development (BSID-III). Psychological Corporation, San Antonio, TX (2006).

19. Griffiths R. Griffiths mental developmental scale- revised: birth to 2 years (GMDS-R). Firenze: Hogrefe; 1996.

20. Armstrong RW. Definition and classification of cerebral palsy. Dev Med Child Neurol. 2007:49:166.

21. Marlow N, Hennessy EM, Bracewell MA, et al. EPICure study group. Motor and executive function at 6 years of age after extremely preterm birth Pediatrics. 2007;120:793-804.

22. Pugliese M, Rossi C, Guidotti I, et al. Preterm birth and developmental problems in infancy and preschool age part II: cognitive, neuropsychological and behavioural outcomes. J Matern Fetal Neonatal Med. 2013;26:1653-7.

23. Pierrat V, Marchand-Martin L, Arnaud C, et al. EPIPAGE-2 writing group Neurodevelopmental outcome at 2 years for preterm children born at 22 to 34 weeks' gestation in France in 2011: EPIPAGE-2 cohort study. BMJ. 2017; 358:j3448

24. Mahoney K, Bajuk B, Oei J, et al. for the NICUS Network. Risk of neurodevelopmental impairment for outborn extremely preterm infants in an Australian regional network. J Matern Fetal Neonatal Med. 2017;30:96-102.

25. Delobel-Ayoub M, Arnaud C, White-Koning M, et al. EPIPAGE Study Group. Behavioral problems and cognitive performance at 5 years of age after very preterm birth: the EPIPAGE study. Pediatrics. 2009;123:1485-92.

26. Romeo DM, Guzzardi S, Ricci D, et al. Longitudinal cognitive assessment in healthy late preterm infants. Eur J Paediatr Neurol. 2012;16:243-7.

27. Serenius F, Källén K, Blennow M, et al. EXPRESS group. Neurodevelopmental outcome in extremely preterm infants at 2.5 years after active perinatal care in Sweden. JAMA. 2013;309:1810-20.

\section{Publisher's Note}

Springer Nature remains neutral with regard to jurisdictional claims in published maps and institutional affiliations.

Ready to submit your research? Choose BMC and benefit from:

- fast, convenient online submission

- thorough peer review by experienced researchers in your field

- rapid publication on acceptance

- support for research data, including large and complex data types

- gold Open Access which fosters wider collaboration and increased citations

- maximum visibility for your research: over $100 \mathrm{M}$ website views per year

At $\mathrm{BMC}$, research is always in progress.

Learn more biomedcentral.com/submissions 